\title{
Increased risk of ischemic stroke and systemic embolism in hyperthyroidism-related atrial fibrillation: A nationwide cohort study
}

Kyu Kim, MD ${ }^{\mathrm{a}, \dagger}$, Pil-Sung Yang, MD ${ }^{\mathrm{b}, \dagger}$, Eunsun Jang ${ }^{\mathrm{a}}$, Hee Tae Yu, MD ${ }^{\mathrm{a}}$, Tae-Hoon Kim, MD ${ }^{\mathrm{a}}$, Jae-Sun Uhm, MD ${ }^{\mathrm{a}}$, Jong-Youn Kim, MD ${ }^{\mathrm{a}}$, Jung-Hoon Sung, MD ${ }^{\mathrm{b}}$, Hui-Nam Pak, MD ${ }^{\mathrm{a}}$, Moon-Hyoung Lee, MD ${ }^{\mathrm{a}}$, Gregory Y.H. Lip, MD ${ }^{\mathrm{a}, \mathrm{c}, \ddagger}$, and Boyoung Joung, MD ${ }^{\mathrm{a}, \sharp}$ England, United Kingdom

Background We aimed to evaluate the long-term risk of ischemic stroke/systemic embolism of hyperthyroidism-related AF.

Methods This retrospective population-based cohort study included records of 1,034,099 atrial fibrillation patients between 2005 and 2016 from the Korean National Health Insurance Service database. After exclusion, we identified 615,724 oral anticoagulation-naïve patients aged $\geq 18$ years with new-onset non-valvular atrial fibrillation, of whom 20,773 had hyperthyroidism-related atrial fibrillation. After 3:1 propensity score matching, ischemic stroke and systemic embolism occurrences were compared between hyperthyroidism-related and non-hyperthyroidism-related ("nonthyroidal") atrial fibrillation patients.

Results After exclusion, we identified 615,724 oral anticoagulation-naïve AF patients of whom 20,773 had hyperthyroidism-related AF. Median follow-up duration was 5.9 years. Hyperthyroidism-related AF patients had significantly higher risks of ischemic stroke and systemic embolism than nonthyroidal AF patients (1.83 vs 1.62 per 100-person year, hazard ratio[HR], $1.13 ; 95 \%$ confidence interval[CI], 1.07 to $1.19 ; P<0.001$ ). This risk was $36 \%$ higher in hyperthyroidismrelated than in nonthyroidal AF patients within 1 year of atrial fibrillation diagnosis $(3.65$ vs 2.67 per 100-person year, $H R$, $1.36 ; 95 \% \mathrm{Cl}, 1.24-1.50 ; P<0.001)$. This difference was also observed in the $\mathrm{CHA}_{2} \mathrm{DS}_{2}-\mathrm{VASc}$ score subgroup analysis. The risk of ischemic stroke and systemic embolism significantly decreased in patients treated for hyperthyroidism (HR, 0.64; $95 \% \mathrm{Cl}, 0.58$ to $0.70 ; P<0.001)$.

Conclusions Hyperthyroidism-related AF patients have high risks of ischemic stroke and systemic embolism like nonthyroidal AF, especially when initially diagnosed. This risk is reduced by treating hyperthyroidism. (Am Heart J 2021;242:123131.)

Atrial fibrillation (AF) is the most common type of cardiac arrhythmia in patients with hyperthyroidism and is observed in $5 \%-15 \%$ of hyperthyroidism cases. ${ }^{1,2}$ Sub-

From the a Division of Cardiology, Department of Internal Medicine, Severance Cardio vascular Hospital, Yonsei University College of Medicine, Seoul, Republic of Korea, b Department of Cardiology, CHA Bundang Medical Center, CHA University, Seongnam, Republic of Korea, 'Liverpool Centre for Cardiovascular Science, University of Liverpool, Liverpool, England, United Kingdom

$\dagger$ The first two authors contributed equally to this work.

$\ddagger$ Joint senior authors

Submitted May 1, 2021; accepted August 28, 2021

Reprint requests: Boyoung Joung, MD, Division of Cardiology, Department of Internal Medicine, Severance Cardiovascular Hospital, Yonsei University College of Medicine,

Reprint requests: Gregory Y.H. Lip, MD, Liverpool Centre for Cardiovascular Science, University of Liverpool, Third Floor, Foundation Building Brownlow Hill Liverpool L69 7TX, United Kingdom

E-mail addresses: gregory.lip@liverpool.ac.uk. $0002-8703$

() Published by access article under the CC BY license
This is an open alsevier
(http://creativecommons.org/licenses/by/4.0/)

https://doi.org/10.1016/i.ahj.2021.08.018 clinical hyperthyroidism and overt thyrotoxicosis are important predisposing factors for $\mathrm{AF}^{3}$ It is well known that $\mathrm{AF}$ is associated with an increased risk of ischemic stroke and systemic embolism (SSE) and that anticoagulation treatment reduces the risk of SSE. ${ }^{4-6}$ However, the risk of SSE in hyperthyroidism-related AF remains unclear. ${ }^{7,8}$

Previous observational studies with small populations have shown a high incidence of ischemic stroke in hyperthyroidism-related $\mathrm{AF}$ patients and claimed the need for anticoagulation treatment. ${ }^{9,10}$ However, in a previous large-scale nationwide cohort study, hyperthyroidism-related AF was not deemed an independent risk factor for stroke. ${ }^{11}$ Thus, previously published clinical guidelines regard hyperthyroidism as a weakly validated risk factor for ischemic stroke in $\mathrm{AF}$ patients. ${ }^{12,13}$ This discrepancy is derived from the nature of hyperthyroidism-related AF. Hyperthyroidism is a curable disease, and its treatment can result in the spontaneous restoration of sinus rhythm in a significant propor- 
tion of patients with hyperthyroidism who present with new-onset $\mathrm{AF} .{ }^{14}$ However, recent studies have shown an increased risk of stroke in "transient" and "resolved" AF patients with various conditions such as postoperative or secondary $\mathrm{AF}^{15,16}$ In the absence of anticoagulation therapy, AF patients may be at a high risk of ischemic stroke. To clarify these uncertainties, we aimed to evaluate the risk of SSE in oral anticoagulation-naive patients with hyperthyroidism-related AF, particularly in relation to time since AF diagnosis and treatment status.

\section{Methods}

\section{Study design and population}

We performed this retrospective nationwide cohort study using the National Health Insurance Service (NHIS) data of Korea. ${ }^{17-20}$ The NHIS is mandatory for all Koreans and includes $97.1 \%$ of the Korean population as mandatory subscribers. This database contains information regarding personal data, medical and pharmaceutical records, inpatient and outpatient service usage, and mortality data. This study was approved by the Institutional Review Board of the Yonsei University Health System (Number 4-2016 - 0179). The need for informed consent was waived. There were 1,034,099 AF patients aged $>18$ years between January 1, 2005 and December 31,2016 . In order to include only newly diagnosed AF patients, we screened for patients who were not diagnosed with AF during a 3-year blanking period from January 2002 to December 2004. As a result, we identified 847,460 newly diagnosed AF patients. AF was defined by International Classification of Disease, Tenth Revision (ICD-10) code I48, as determined in 1hospitalization or 2outpatient visits. To evaluate the accuracy of our definition of AF, we conducted a validation study with 628 randomly chosen patients from 2 hospitals who had ICD10 code I48 and underwent electrocardiography. Their electrocardiograms were reviewed by two physicians. Patients were diagnosed with AF with a positive predictive value of $94.1 \% .^{17-20}$

Hyperthyroidism was defined by ICD-10 code E05. In a validation study with 189 randomly chosen patients with ICD-10 code E05, hyperthyroidism diagnosis had a positive predictive value of $94.7 \%$. Hyperthyroidism-related AF was defined as a hyperthyroidism diagnosis claim occurring 30 days before or after AF diagnosis. The index date was defined as the date of AF diagnosis. We excluded patients with valvular AF (including diagnosis of mitral stenosis or prosthetic heart valves, or insurance claims for valve replacement or valvuloplasty), patients with insurance claim occurred at the same date of $\mathrm{AF}$ diagnosis, and thyroid cancer and also those prescribed oral anticoagulants before cohort enrollment. After exclusion, there were 615,724 oral anticoagulation-naive non-valvular AF patients, of whom 20,773 were classified as hyperthyroidism-related AF patients. The rest were as-

\section{Figure 1}

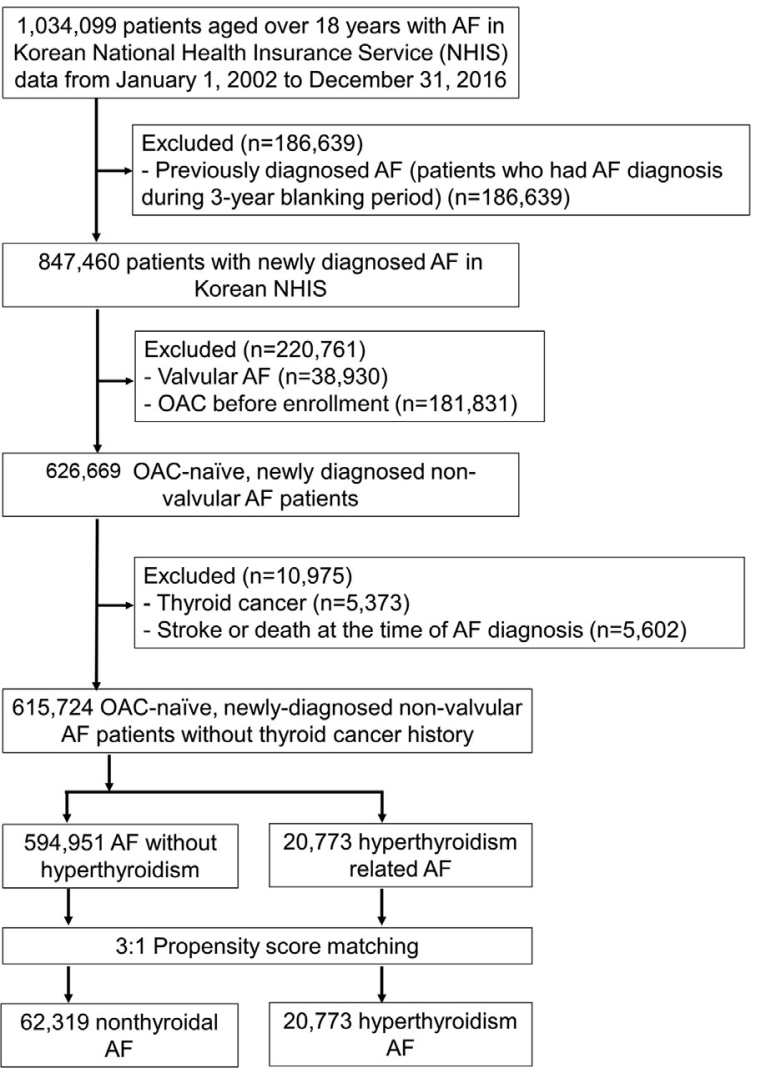

Flowchart of the study population selection and analysis.

signed to the control group, which was defined as the nonthyroidal AF group. All study groups were matched via 1:3 propensity score matching. Details are presented in (Figure 1).

\section{Covariates and outcome data}

We obtained information on select comorbid conditions from inpatient and outpatient hospital diagnoses. Baseline comorbidities were defined using medical claims and prescription medication data prior to the index date. To ensure diagnostic accuracy, patients were considered to have comorbidities when the condition was a discharge diagnosis or was confirmed at least twice in an outpatient setting. This was similar to the methods reported in previous studies (eTable 1 ). ${ }^{17-20}$

Patients' first ischemic stroke or systemic embolism was assessed. The definitions of clinical outcomes are presented in S1 Table. The accuracy of ischemic stroke diagnoses based on the NHIS claim data was previously validated. ${ }^{17-20}$ Patients were followed up from the index date until the occurrence of the study outcome, initiation of oral anticoagulant, or end of the follow-up pe- 
Table I. Comparison of baseline characteristics between atrial fibrillation patients with and without hyperthyroidism

\begin{tabular}{|c|c|c|c|c|c|c|}
\hline \multirow[t]{2}{*}{ Characteristics } & \multicolumn{3}{|c|}{ Before propensity score matching } & \multicolumn{3}{|c|}{ Propensity score-matched cohort } \\
\hline & $\begin{array}{l}\text { Nonthyroidal AF } \\
(n=594,951)\end{array}$ & $\begin{array}{l}\text { Hyperthyroidism AF } \\
(n=20,773)\end{array}$ & SMD & $\begin{array}{l}\text { Nonthyroidal AF } \\
(n=62,319)\end{array}$ & $\begin{array}{l}\text { Hyperthyroidism AF } \\
(n=20,773)\end{array}$ & SMD \\
\hline Age (years) & $67.0(55.0-76.0)$ & $61.0(48.0-72.0)$ & 0.312 & $61.0(48.0-72.0)$ & $61.0(48.0-72.0)$ & 0.003 \\
\hline$\geq 65$ & $330101(55.5 \%)$ & $8693(41.8 \%)$ & 0.275 & $26937(43.2 \%)$ & $8693(41.8 \%)$ & 0.028 \\
\hline$\geq 75$ & $171402(28.8 \%)$ & $4086(19.7 \%)$ & 0.214 & $12774(20.5 \%)$ & $4086(19.7 \%)$ & 0.021 \\
\hline Female & $275249(46.3 \%)$ & $10833(52.1 \%)$ & 0.118 & $32577(52.3 \%)$ & $10833(52.1 \%)$ & 0.003 \\
\hline Hypertension & $407764(68.5 \%)$ & $11502(55.4 \%)$ & 0.274 & $34202(54.9 \%)$ & 11502 (55.4\%) & 0.010 \\
\hline Diabetes mellitus & $136656(23.0 \%)$ & $3443(16.6 \%)$ & 0.161 & $10188(16.3 \%)$ & $3443(16.6 \%)$ & 0.006 \\
\hline Heart failure & $139771(23.5 \%)$ & $3466(16.7 \%)$ & 0.171 & $10365(16.6 \%)$ & $3466(16.7 \%)$ & 0.001 \\
\hline Ischemic stroke or TIA & $120541(20.3 \%)$ & $2603(12.5 \%)$ & 0.210 & $7700(12.4 \%)$ & $2603(12.5 \%)$ & 0.005 \\
\hline Vascular disease & $97605(16.4 \%)$ & $2603(12.5 \%)$ & 0.178 & $6465(10.4 \%)$ & $2157(10.4 \%)$ & 0.000 \\
\hline $\mathrm{CHA}_{2} \mathrm{DS}_{2}$-VASc score & $2.0(1.0-4.0)$ & $2.0(1.0-4.0)$ & 0.313 & $2.0(1.0-4.0)$ & $2.0(1.0-4.0)$ & 0.006 \\
\hline$<2$ & $175148(29.4 \%)$ & $8888(42.8 \%)$ & 0.281 & $26077(41.8 \%)$ & $8888(42.8 \%)$ & 0.019 \\
\hline$\geq 2$ & $275249(46.3 \%)$ & $11885(57.2 \%)$ & 0.281 & $36242(58.2 \%)$ & $11885(57.2 \%)$ & 0.019 \\
\hline History of major bleeding & $81247(13.7 \%)$ & $1788(8.6 \%)$ & 0.161 & $5392(8.7 \%)$ & $1788(8.6 \%)$ & 0.002 \\
\hline Dyslipidemia & $339291(57.0 \%)$ & $9555(46.0 \%)$ & 0.222 & $28533(45.8 \%)$ & $9555(46.0 \%)$ & 0.004 \\
\hline COPD & $95327(16.0 \%)$ & $2211(10.6 \%)$ & 0.159 & $6679(10.7 \%)$ & $2211(10.6 \%)$ & 0.002 \\
\hline Chronic kidney disease & $35715(6.0 \%)$ & $688(3.3 \%)$ & 0.128 & $2046(3.3 \%)$ & $688(3.3 \%)$ & 0.002 \\
\hline End-stage renal disease & 7707 (1.3\%) & $115(0.6 \%)$ & 0.078 & $359(0.6 \%)$ & $115(0.6 \%)$ & 0.003 \\
\hline History of Liver disease & $227108(38.2 \%)$ & $6419(30.9 \%)$ & 0.153 & $18838(30.2 \%)$ & $6419(30.9 \%)$ & 0.015 \\
\hline History of malignant neoplasm & $136052(22.9 \%)$ & $2850(13.7 \%)$ & 0.238 & $8552(13.7 \%)$ & $2850(13.7 \%)$ & 0.000 \\
\hline Aspirin & $211344(35.5 \%)$ & $4800(23.1 \%)$ & 0.275 & $14544(23.3 \%)$ & $4800(23.1 \%)$ & 0.005 \\
\hline P2Y12 inhibitor & $63178(10.6 \%)$ & $945(4.5 \%)$ & 0.231 & $2954(4.7 \%)$ & $945(4.5 \%)$ & 0.009 \\
\hline ACE or ARB & $216667(36.4 \%)$ & $5136(24.7 \%)$ & 0.256 & $15267(24.5 \%)$ & $5136(24.7 \%)$ & 0.005 \\
\hline Beta blocker & $186598(31.4 \%)$ & $4250(20.5 \%)$ & 0.251 & $12822(20.6 \%)$ & $4250(20.5 \%)$ & 0.003 \\
\hline CCB (DHP) & $217256(36.5 \%)$ & $5741(27.6 \%)$ & 0.191 & 17097 (27.4\%) & $5741(27.6 \%)$ & 0.005 \\
\hline CCB (Non-DHP) & $31199(5.2 \%)$ & $580(2.8 \%)$ & 0.125 & $1831(2.9 \%)$ & $580(2.8 \%)$ & 0.009 \\
\hline AAD (Class Ic) & $5417(0.9 \%)$ & $46(0.2 \%)$ & 0.092 & $190(0.3 \%)$ & $46(0.2 \%)$ & 0.016 \\
\hline AAD (Class III) & $5781(1.0 \%)$ & $78(0.4 \%)$ & 0.073 & $236(0.4 \%)$ & $78(0.4 \%)$ & 0.001 \\
\hline Diuretics & $223982(37.6 \%)$ & $5508(26.5 \%)$ & 0.240 & $16415(26.3 \%)$ & $5508(26.5 \%)$ & 0.004 \\
\hline Digoxin & $32332(5.4 \%)$ & $575(2.8 \%)$ & 0.135 & $1788(2.9 \%)$ & $575(2.8 \%)$ & 0.006 \\
\hline Statin & $147789(24.8 \%)$ & $3193(15.4 \%)$ & 0.238 & 9575 (15.4\%) & 3193 (15.4\%) & 0.000 \\
\hline
\end{tabular}

Values are presented as median (IQR) or $n(\%)$.

$A A D$, antiarrhythmic drug; $A C E$, angiotensin converting enzyme; $A F$, atrial fibrillation; $A R B$, angiotensin II receptor blocker; $C C B$, calcium channel blocker; $C O P D$, chronic obstructive pulmonary disease; DHP, dihydrodipine; IQR, interquartile range; SMD, standardized mean difference; TIA, transient ischemic attack.

riod, whichever occurred first. Each end point was analyzed independently of the others without being censored. We analyzed the risk of SSE and incidence rate difference between groups in different time periods (within 1 year of AF diagnosis and overall study periods). Additionally, we performed subgroup analyses based on patients' covariates and hyperthyroidism treatment status. Treated hyperthyroidism was defined as hyperthyroidism treated with antithyroid drugs for at least 1 month since hyperthyroidism diagnosis. Treated intractable hyperthyroidism was defined as hyperthyroidism treated with radioactive iodine or thyroidectomy.

\section{Statistical analyses}

Data are presented as median (interquartile range [IQR]) for continuous variables and number (percentage) for categorical variables. We constructed a 3:1 propensity score model based on multiple logistic regression analysis that included the following variables: age; sex; components of the $\mathrm{CHA}_{2} \mathrm{DS}_{2}$-VASc score (hypertension, diabetes mellitus, heart failure, prior ischemic stroke or transient ischemic attack [TIA] events, and vascular disease); patients' dyslipidemia, chronic obstructive pulmonary disease, chronic kidney disease, and liver disease status; history of major bleeding; previous cancer diagnoses; and prescribed medication at enrollment. Matching was performed using the nearest-neighbor matching method, in which patients were matched on the logit of the propensity score using a caliper width of 0.1 of the standard deviation. Standardized mean differences were estimated to assess pre- and post-match balance. We used Aalen-Johansen analysis to construct cumulative incidence curves with competing risk as death. Poisson distribution to calculate SSE incidence rates and incidence rate difference (IRD) with 95\% confidence intervals (CIs) and, and Cox regression to calculate hazard ratios (HRs) with 95\% CIs. We analyzed clinical outcomes within 1 year of enrollment and overall study periods. We computed time-dependent hazard rates and HRs using generalized survival models which were created using the stpm2 function in the "rstpm2" $\mathrm{R}$ package. We identified sufficient overlaps of propensity scores between groups, 
Table II. Incidence rates and hazard ratios of ischemic stroke in hyperthyroidism-related atrial fibrillation patients

Nonthyroidal AF $(n=62,319)$

Hyperthyroidism AF $(n=20,773)$

SSE

Events

Person years

Incidence rate $(95 \% \mathrm{Cl})^{\dagger}$

Absolute incidence rate difference $(95 \% \mathrm{Cl})$

Hazard ratio $(95 \% \mathrm{Cl})^{\ddagger}, P$ value

Within 1 year

Events

Person years

Incidence rate $(95 \% \mathrm{Cl})$

Absolute incidence rate difference $(95 \% \mathrm{Cl})$

Hazard ratio $(95 \% \mathrm{Cl}), P$ value

4,980
307937.1
$1.62(1.57$ to 1.66$)$
1 (Reference)
1,429
53555.99
2.67 (2.53 to 2.81 )
1 (Reference)

1,806

98488.17

1.83 (1.75 to 1.92$)$

$0.22(0.12$ to 0.31$)$

$1.13(1.07$ to 1.19$),<0.001$

633

17323.62

3.65 (3.38 to 3.95$)$

$0.99(0.67$ to 1.30$)$

$1.36(1.24$ to 1.50$),<0.001$

Values are presented as $n(\%)$, unless stated otherwise

$\mathrm{AF}$, atrial fibrillation; $95 \% \mathrm{Cl}, 95 \%$ confidence interval; SSE, stroke and systemic embolism

${ }^{\dagger}$ Per 100 person-years.

${ }^{\ddagger} \mathrm{HR}$ adjusted for age, sex, hypertension, diabetes mellitus, congestive heart failure, major bleeding history, vascular disease, history of stroke/transient ischemic attack, dyslipidemia, Charlson comorbidity index category (chronic obstructive pulmonary disease, peptic ulcer disease, liver disease, chronic kidney disease, dementia, human immunodeficiency virus infection, and malignancy), and hypertrophic cardiomyopathy.

which represents the existence of equipoise between the 2group (eFigure 1). ${ }^{21}$ We also performed the following sensitivity analyses to make our study findings more robust. 1) We used a Cox multivariable regression model to analyze clinical outcomes of subjects before propensity score matching. This model contained all the variables listed in (Table I - II 2) We also used the inverse probability of treatment weighting method for analyzing clinical outcomes. 3) We assessed the risk of SSE without censoring at the date of oral anticoagulant initiation. Statistical analyses were conducted using SAS version 9.3 (SAS Institute, Cary, NC, USA) and R version 3.3.2 (The R foundation, www.R-project.org).

\section{Results}

\section{Baseline characteristics}

Patients' baseline characteristics are presented in (Table I ). Of the 615,724 oral anticoagulation-naive nonvalvular AF patients, 20,773 (3.4\%) had hyperthyroidismrelated AF. After 3:1 propensity score matching, all groups had well-balanced baseline characteristics (Table I ). The median age and $\mathrm{CHA}_{2} \mathrm{DS}_{2}$-VASc score of the study subjects were 61.0 (IQR, 48.0 - 72.0) and 2.0 (IQR, 1.0 - 4.0), respectively (Table I ). The median follow-up duration was 5.9 years. During follow-up periods, $30.7 \%$ patients were prescribed oral anticoagulants and censored.

\section{Risk of ischemic SSE}

During the study period, 1,806 and 4,980 SSE events occurred in the hyperthyroidism-related AF and nonthyroidal AF groups, respectively. The incidence rate of SSE events was 1.83 per 100 person-years (95\% CI, 1.75 to 1.92 ) in the hyperthyroidism-related AF group and 1.62 per 100 person-years (95\% CI, 1.57 to 1.66 ) in the nonthyroidal AF group (Table II ). Additionally, the hyperthyroidism-related AF group had a significantly higher cumulative SSE incidence rate than the nonthyroidal AF group (log-rank, $P<0.001$ ) (Figure 2). The hyperthyroidism-related AF group also had a significantly higher risk of SSE than the nonthyroidal AF group (HR, 1.13; 95\% CI, 1.07 to $1.19 ; \boldsymbol{P}<0.001$ ) (Table II ).

Time-dependent changes in the risk of SSE

There were higher incidences of SSE within 1 year of AF diagnosis in both study groups. We observed $633(35.0 \%)$ SSE events in the hyperthyroidism-related AF group and 1,489 (29.9\%) SSE events in the nonthyroidal AF group. The risk of SSE was 36\% higher in the hyperthyroidism-related AF group than in the nonthyroidal AF group within 1 year of AF diagnosis (HR, 1.36; 95\% CI, 1.24 to 1.50 , IRD, $0.99 ; 95 \% \mathrm{CI}, 0.67$ to $1.30 ; P$ $<0.001$ ), but IRD decreased over time (IRD, 0.22; $95 \%$ CI 0.12 to 0.31 ) (Table II , Figure 2).

The time-dependent hazard rates of SSE are presented in (Figure 3A). Within 1 year of AF diagnosis, SSE hazard rates were higher in the hyperthyroidism-related $\mathrm{AF}$ than in the nonthyroidal AF group. Among the 1,806 SSE events that occurred in the hyperthyroidism-related AF group, 633 (35.0\%) occurred within 1 year of AF diagnosis. Beyond 1 year of AF diagnosis, the difference in hazards became statistically insignificant between the two groups. The time-dependent HRs are shown in (Figure 3B).

\section{Risk of SSE according to $\mathrm{CHA}_{2} \mathrm{DS}_{2}$-VASc score}

SSE incidence rates based on $\mathrm{CHA}_{2} \mathrm{DS}_{2}$-VASc scores are presented in (Figure 4). During the study period, the annual incidence of SSE exceeded 2 per 100 person-years in hyperthyroidism-related AF patients with a $\mathrm{CHA}_{2} \mathrm{DS}_{2^{-}}$ VASc score $\geq 3$. Moreover, the hyperthyroidism-related 


\section{Figure 2}

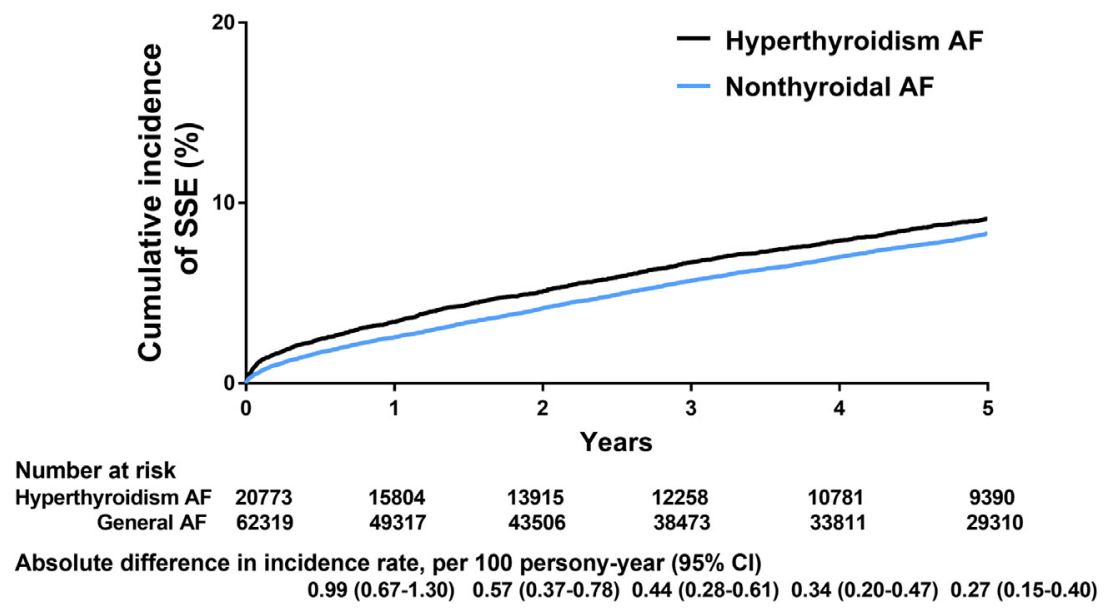

The cumulative incidence SSE and incidence rate difference in AF patients with and without hyperthyroidism. AF, atrial fibrillation; SSE, ischemic stroke and systemic embolism

\section{Figure 3}

A

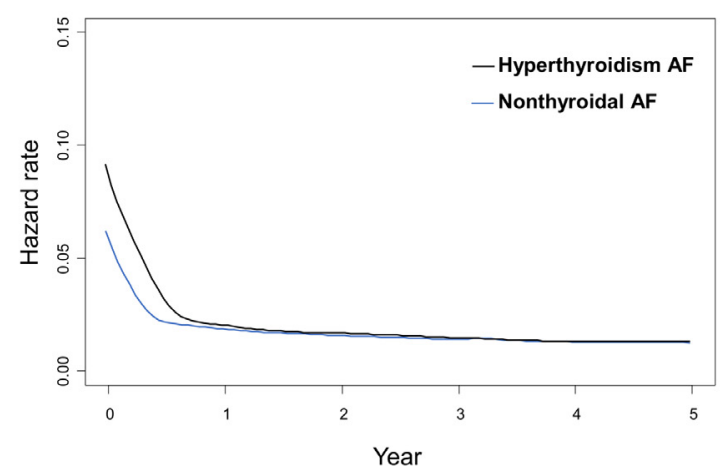

B Time dependent hazard ratio

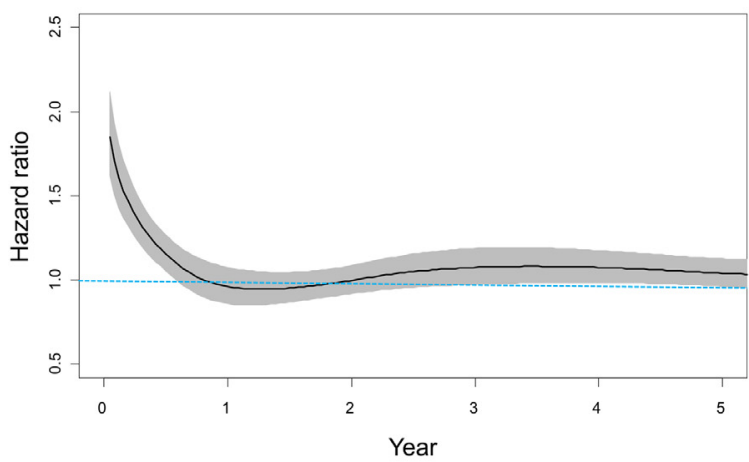

Time-dependent hazard rates $A$, and hazard ratios $B$, of the study groups.

AF group had higher SSE incidence rates than the nonthyroidal AF group with respect to patients with a $\mathrm{CHA}_{2} \mathrm{DS}_{2-}$ VASc score $>5$. Within 1 year of AF diagnosis, the incidence of SSE exceeded 2 per 100 person-year in hyperthyroidism-related AF patients with a $\mathrm{CHA}_{2} \mathrm{DS}_{2^{-}}$ VASc score $\geq 2$. In patients with hyperthyroidism-related AF group and lower SSE risk $\left(\mathrm{CHA}_{2} \mathrm{DS}_{2}\right.$-VASc score $\left.<2\right)$, SSE incidence was 0.69 (95\% CI, 0.62 to 0.77$)$ per 100 person-year.

\section{Subgroup analysis}

The hyperthyroidism-related AF group had a higher risk of SSE than the nonthyroidal AF group in our subgroup analyses regarding age, comorbidities (hypertension and heart failure status), sex, prior ischemic stroke or TIA events, and $\mathrm{CHA}_{2} \mathrm{DS}_{2}$-VASc score subgroups (all $P$ for interaction $>0.05$ ) (Figure 5). The risk of SSE in the treated hyperthyroidism (HR, $0.64 ; 95 \%$ CI, 0.58 to 0.70 ; $P$ for interaction $<0.001)$ and treated intractable hyperthyroidism subgroups (HR, $0.45 ; 95 \% \mathrm{CI}, 0.28$ to 0.73 ; $P$ for interaction $<0.001)$ was significantly lower than that in the nonthyroidal AF group. The risk of SSE decreased in the treated hyperthyroidism subgroup within 1 year of hyperthyroidism diagnosis. The untreated hyperthyroidism subgroup had a significantly higher risk of SSE than the nonthyroidal AF group (HR, 1.46; 95\% CI, 1.35 to 1.55 ) (Figure 5). 


\section{Figure 4}

A

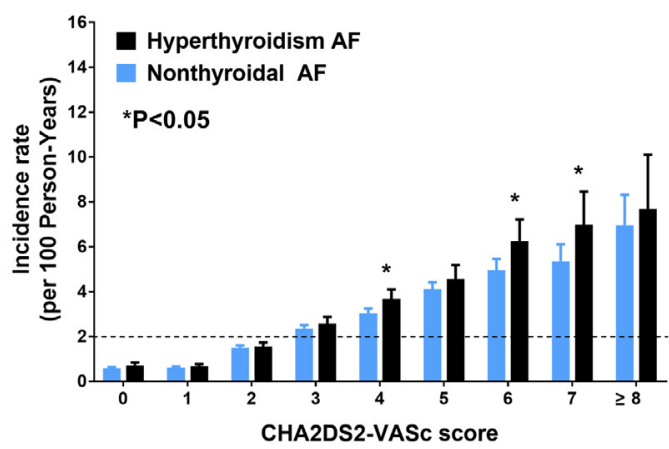

Within 1 year from AF diagnosis

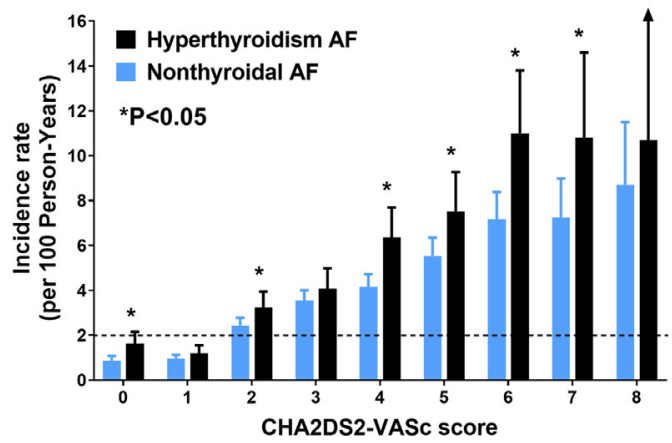

Incidence rate of SSE in AF patients with and without hyperthyroidism stratified by CHA2DS2-VASc score. A, overall follow-up duration; B, within 1 year from AF diagnosis. ${ }^{*} P<0.05$

Figure 5
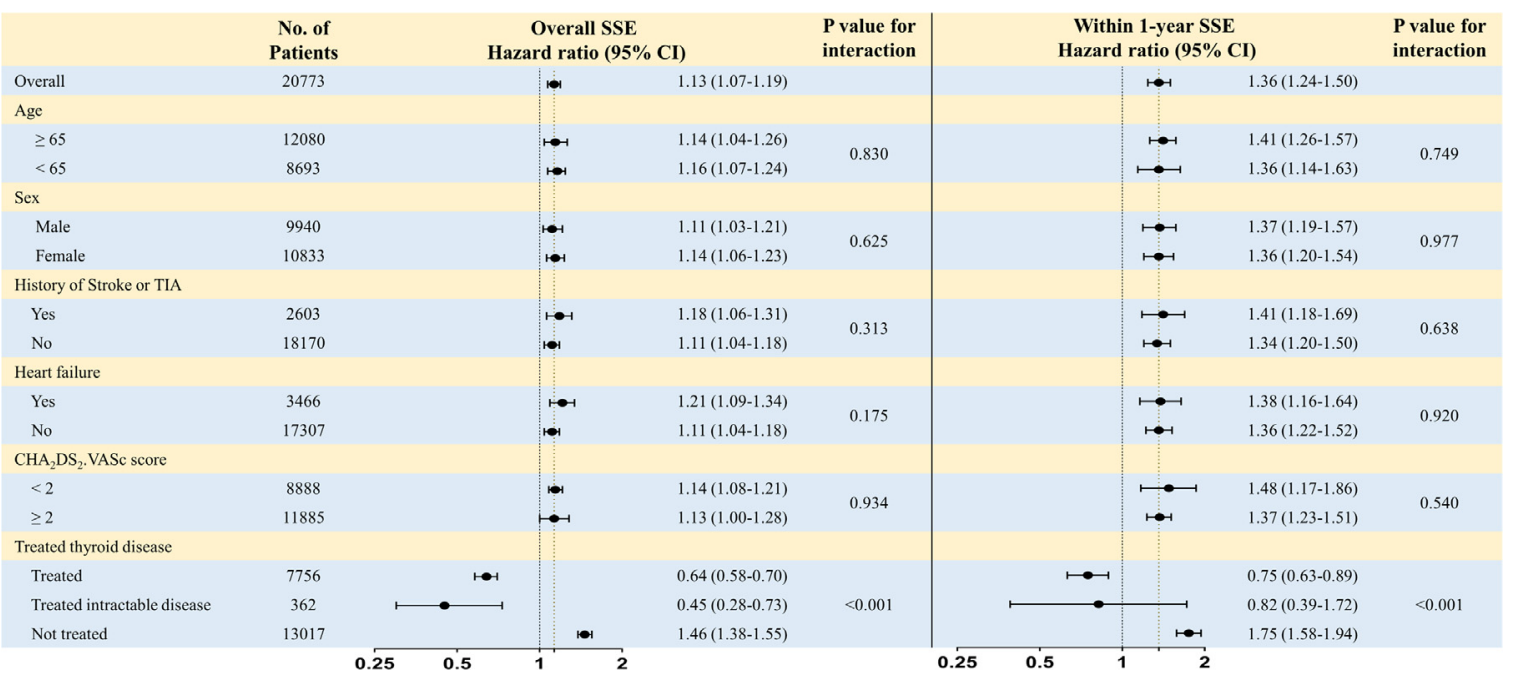

Hazard ratios of ischemic stroke and systemic embolism in different subgroups.

\section{Sensitivity analysis}

For all subjects, before propensity score matching, hyperthyroidism was significantly associated with the increased risk of SSE, as determined based on the following adjusted HRs: 1.13 (95\% CI, 1.08 to $1.18 ; P<0.001)$ during the whole study period, 1.36 (95\% CI, 1.26 to 1.48 ; $P<0.001)$ within 1 year of AF diagnosis, and $1.04(95 \%$ CI, 0.98 to $1.10 ; P=0.205$ ) beyond 1 year of AF diagnosis. In the inverse probability of treatment-weighted cohort, the results were still similar to the main result. The assessed SSE risk without censoring at the date of oral anticoagulant initiation was consistent with primary findings (eTable II ).

\section{Discussion}

In this large-scale retrospective cohort study, hyperthyroidism-related AF patients showed a higher incidence of SSE than nonthyroidal AF patients within 1 year of AF diagnosis, but incidence of SSE became similar to that of nonthyroidal AF over time. This trend remained consistent in all ranges of $\mathrm{CHA}_{2} \mathrm{DS}_{2}$-VASc scores. After hyperthyroidism treatment, the incidence rate of SSE significantly decreased and was lower in hyperthyroidism-related $\mathrm{AF}$ patients than in nonthyroidal AF patients. Our findings suggest that hyperthyroidismrelated $\mathrm{AF}$ patients might have higher risks of ischemic stroke and systemic embolism, especially when initially 
diagnosed. This risk might be reduced by treating hyperthyroidism. However, further prospective study would be needed.

\section{Increased risk of SSE in hyperthyroidism-related AF}

Hyperthyroidism is a common endocrinologic disorder that can exacerbate pre-existing cardiac disease and cause de novo cardiovascular abnormalities, including $\mathrm{AF}$, heart failure, and cardiovascular disease. ${ }^{22,23}$ Despite the close associations between AF and hyperthyroidism and ischemic stroke, whether hyperthyroidismrelated $\mathrm{AF}$ results in a higher risk of ischemic stroke than nonthyroidal AF remains controversial. Therefore, hyperthyroidism-related $\mathrm{AF}$ is not regarded as a major risk factor for ischemic stroke in current guidelines. ${ }^{13}$ Only Canadian Cardiovascular Society guidelines suggested anticoagulation during thyrotoxic state with low quality evidence. ${ }^{24}$ Prior observational studies have reported that the risk of SSE was higher in hyperthyroidism-related AF patients than in nonthyroidal AF patients. Moreover, a prospective observational study of 480 patients by Siu et al. revealed that hyperthyroidism-related AF patients had a higher risk of stroke than nonthyroidal AF patients. $^{9,10}$ Alternatively, a retrospective cohort study using Swedish nationwide cohort data reported that thyroid disease was not an independent risk factor for stroke (HR, 0.96 ; 95\% CI, 0.73 to 1.25 ). However, while this study analyzed 182,678 AF patients without oral anticoagulation, only 55 ischemic stroke events occurred in hyperthyroidism-related AF patients. Moreover, the number of such patients was not presented. ${ }^{11}$

In our study, we analyzed 615,724 oral anticoagulationnaïve non-valvular AF patients, which included 20,773 patients with hyperthyroidism. To our knowledge, our study is the largest study that investigates the risk of stroke in hyperthyroidism-related AF.

The mechanism(s) for an increased risk of thromboembolism associated with new-onset AF in patients with hyperthyroidism remains unclear. The high risk of stroke in hyperthyroidism-related AF may be derived from hemostatic changes in thyroid disease. ${ }^{25,26} \mathrm{Hy}$ pothyroid and hyperthyroid statuses are associated with abnormal hemostasis, whereby the coagulation factor turnover rate and fibrinogen levels increase. ${ }^{26}$ Hypercoagulability in new-onset non-valvular AF patients may also play a role in this abnormal hemostasis. ${ }^{27}$ Moreover, hyperthyroidism is associated with hypercoagulability. Therefore, the presence of both $\mathrm{AF}$ and hyperthyroidism may synergistically increase thromboembolic risk. ${ }^{28}$

Increased risk of SSE within 1 year of AF diagnosis and reduced risk after treatment

Our study shows an increased risk of SSE within 1 year of AF diagnosis, concurring with results of the study of GARFIELD-AF data that showed that the highest number of clinical events occurred during the first 4 months of follow-up, ${ }^{27}$ results reported by Siu et al. where the majority of ischemic strokes $(>70 \%)$ occurred within the first 30 days of presentation, ${ }^{9}$ and results reported by Dekkers et al. ${ }^{23}$ who showed an increased risk of death and cardiovascular events in the initial months of hyperthyroidism diagnosis. ${ }^{27,28}$ Half of our hyperthyroidismrelated AF patients did not receive hyperthyroidism treatment, possibly because some had subclinical hyperthyroidism, which is also classified as ICD code E05. There have been several studies on the association between subclinical hyperthyroidism and $\mathrm{AF}^{22,29}$ Despite this, patients treated for hyperthyroidism had a significantly lower risk of SSE than nonthyroidal AF patients, even in intractable cases. Insufficient hyperthyroidism treatment has also been associated with an increased cardiovascular risk. ${ }^{30}$ Unfortunately, the rate of sinus conversion was not available in this study. However, there was a data about spontaneous conversion to sinus rhythm after hyperthyroidism treatment can result in decreased AF burden and a lower risk of SSE. ${ }^{31}$ In this study, there was a residual SSE risk during entire follow-up duration. In this study, there was a residual SSE risk during entire follow-up duration. However, the incidence rate of SSE was not significant in subgroup with low CHA2DS2-VASc score under 2, who do not need for anticoagulation in current guidelines. ${ }^{12,13}$ These finding suggest anticoagulation might be not required in patients with hyperthyroidism related AF and CHA2DS2-VASc score under 2.

When the study population was classified according to the $\mathrm{CHA}_{2} \mathrm{DS}_{2}$-VASc score, SSE incidence rates were around 2 per 100 person-years in patients whose $\mathrm{CHA}_{2} \mathrm{DS}_{2}$-VASc score was $\geq 2$. Our results are consistent with those of our previous study, which investigated risk factors for stroke and the application of the $\mathrm{CHA}_{2} \mathrm{DS}_{2}$ VASc score in the Korean AF population. ${ }^{32}$

\section{Clinical implications}

In contrast to current guidelines, ${ }^{12,13}$ we demonstrated the increased risk of SSE in hyperthyroidism-related AF patients compared with that in nonthyroidal AF patients, especially within the first year of AF diagnosis. The risk became similar to that of nonthyroidal AF patients over time. Therefore, stroke prevention via oral anticoagulation may be required in hyperthyroidism-related AF patients. Furthermore, hyperthyroidism treatment may reduce such patients' risk of stroke.

\section{Study limitations}

Our study had several limitations. First, AF diagnoses and adverse events were based on the ICD-10 codes registered by the physicians responsible for patient care. However, the accuracy of AF and adverse event diagnoses in the Korean NHIS database were previously validated. ${ }^{18-21}$ Second, data on different AF types (paroxysmal and nonparoxysmal) were not available. Third, our study was based on Korean NHIS data, and we had 
no information about thyroid function tests (TSH, T3, T4), autoimmunity status (TRAb, anti-TPO, anti-Tg), UL and scintigraph findings. We also did not have data for hyperthyroidism causes (offending drug such as amiodarone, glucocorticoids, or oral contraceptives), and the exact incidence of subclinical hyperthyroidism. So, we were not able to clarify the treatment response for hyperthyroidism. Fourth, definition of intractable hyperthyroidism was ambiguous. Some patients might be received radioiodine or thyroidectomy because of contraindication for antithyroid drug. Fifth, we did not have exact data of returning sinus rhythm after hyperthyroidism treatment. Sixth, as the present study included only OAC naive patients, we were not able to prove the benefit of anticoagulation in this population. Seventh, the definition of comorbidities in this study might ensure a high positive predictive value, but there were concerns for low sensitivity. Finally, we may have missed confounders that affected clinical events such as obesity, smoking or alcohol consumption. Despite these limitations, this study included evaluated longitudinal data from almost the entire Korean adult population, and to our knowledge, our study is the largest study to investigate the risk of stroke in hyperthyroidism-related AF patients.

\section{Conclusion}

The risk of SSE was similar in hyperthyroidismrelated AF and nonthyroidal AF group. Within 1 year of AF diagnosis, the risk of SSE was much higher in hyperthyroidism-related AF group than nonthyroidal AF group. This risk was seemed to be reduced by treating hyperthyroidism. Hyperthyroidism-related AF patients have a high risk of stroke when initially diagnosed with AF and thus require regular follow-ups with appropriate anticoagulation strategy and prompt treatment for hyperthyroidism.

\section{Funding}

This research was supported by a grant of PatientCentered Clinical Research Coordinating Center (PACEN) funded by the Ministry of Health \& Welfare, Republic of Korea (grant number : HI19C0481, HC19C013, HI15C1200) and Korea-UK grant (HI19C0622). The funding sources were not involved in the study design and data collection, analysis, or interpretation.

\section{Conflict of interest}

Dr. Gregory Y.H. Lip has served as a consultant for Bayer/Janssen, BMS/Pfizer, Biotronik, Medtronic, Boehringer Ingelheim, Novartis, Verseon, and DaiichiSankyo and as a speaker for Bayer, BMS/Pfizer, Medtronic, Boehringer Ingelheim, and Daiichi-Sankyo. No fees were directly received personally. Dr. Boyoung Joung has served as a speaker for Bayer, BMS/Pfizer, Medtronic, and
Daiichi-Sankyo, and has received research funding from Medtronic and Abbott. No fees were directly received personally. The other authors have no conflict of interest.

\section{Author contributions}

Conceptualization: Pil-Sung Yang, Boyoung Joung, Gregory Y.H. Lip.

Data curation: Pil-Sung Yang, Eunsun Jang, Hee Tae Yu.

Formal analysis: Kyu Kim, Boyoung Joung.

Funding acquisition: Boyoung Joung, Gregory Y.H. Lip.

Investigation: Jae-Sun Uhm, Jong-Youn Kim, JungHoon Sung, Hui-Nam Pak, Moon-Hyoung Lee.

Methodology: Kyu Kim, Pil-Sung Yang, Boyoung Joung, Gregory Y.H. Lip.

Supervision: Jung-Hoon Sung, Hui-Nam Pak, MoonHyoung Lee, Boyoung Joung, Gregory Y.H. Lip.

Visualization: Kyu Kim.

Validation: Kyu Kim, Pil-Sung Yang.

Writing - original draft: Kyu Kim, Pil-Sung Yang, Boyoung Joung.

Writing - review \& editing: Kyu Kim, Pil-Sung Yang, Hee Tae Yu, Tae-Hoon Kim, Jae-Sun Uhm, Jong-Youn Kim, Jung-Hoon Sung, Hui-Nam Pak, Moon-Hyoung Lee, Gregory Y.H. Lip, Boyoung Joung.

\section{Additional information}

The e-Figures can be found in the Supplemental Materials section of the online article.

\section{Supplementary materials}

Supplementary material associated with this article can be found, in the online version, at doi:10.1016/j.ahj. 2021.08.018.

\section{References}

1. Frost L, Vestergaard P, Mosekilde L. Hyperthyroidism and risk of atrial fibrillation or flutter: a population-based study. Arch Intern Med 2004;164:1675-8.

2. Klein I, Danzi S. Thyroid disease and the heart. Circulation 2007; 1 16:1725-35.

3. Biondi B, Cooper DS. Subclinical Hyperthyroidism. N Engl J Med 2018;378:2411-19

4. Joung B, Lee JM, Lee KH, et al. 2018 Korean guideline of atrial fibrillation management. Korean Circ J 2018;48:1033-80.

5. Lip G, Freedman B, De Caterina R, et al. Stroke prevention in atrial fibrillation: past, present and future. comparing the guidelines and practical decision-making. Thromb Haemost 2017;117:1230-9.

6. Lip GYH, Banerjee A, Boriani G, et al. Antithrombotic therapy for atrial fibrillation: chest guideline and expert panel report. Chest 2018;154:1121-201

7. Squizzato A, Gerdes VE, Brandjes DP, et al. Thyroid diseases and cerebrovascular disease. Stroke 2005;36:2302-10. 
8. Friberg L, Benson L, Rosenqvist $M$, et al. Assessment of female sex as a risk factor in atrial fibrillation in Sweden: nationwide retrospective cohort study. BM 2012;344:e3522.

9. Siu CW, Pong $V$, Zhang $X$, et al. Risk of ischemic stroke after new-onset atrial fibrillation in patients with hyperthyroidism. Heart Rhythm 2009;6:169-73.

10. Bar-Sela S, Ehrenfeld M, Eliakim M. Arterial embolism in thyrotoxicosis with atrial fibrillation. Arch Intern Med 1981;141:1191-2.

11. Friberg L, Rosenqvist M, Lip GY. Evaluation of risk stratification schemes for ischaemic stroke and bleeding in 182678 patients with atrial fibrillation: the swedish atrial Fibrillation cohort study. Eur Heart J 2012;33:1500-10.

12. January CT, Wann LS, Calkins H, et al. 2019 AHA/ACC/HRS Focused Update of the 2014 AHA/ACC/HRS guideline for the management of patients with atrial fibrillation: a report of the American College of Cardiology/American Heart Association Task Force on Clinical Practice Guidelines and the Heart Rhythm Society. J Am Coll Cardiol 2019;74:104-32.

13. Hindricks G, Potpara T, Dagres N, et al. 2020 ESC Guidelines for the diagnosis and management of atrial fibrillation developed in collaboration with the European Association for Cardio-Thoracic Surgery (EACTS): the task force for the diagnosis and management of atrial fibrillation of the European Society of Cardiology (ESC) Developed with the special contribution of the European Heart Rhythm Association (EHRA) of the ESC. Eur Heart J $2021 ; 42: 373-498$.

14. Smith TJ, Hegedus L. Graves' Disease. N Engl J Med 2016;375:1552-65.

15. Kim K, Yang PS, Jang E, et al. Long-term impact of newly diagnosed atrial fibrillation during critical care: a south korean nationwide cohort study. Chest 2019;156:518-28.

16. Adderley NJ, Nirantharakumar K, Marshall T. Risk of stroke and transient ischaemic attack in patients with a diagnosis of resolved atrial fibrillation: retrospective cohort studies. BM 2018;361:k1717

17. Kim D, Yang PS, Kim TH, et al. Ideal blood pressure in patients with atrial fibrillation. J Am Coll Cardiol 2018;72:1233-45.

18. Kim TH, Yang PS, Yu HT, et al. Effect of hypertension duration and blood pressure level on ischaemic stroke risk in atrial fibrillation: nationwide data covering the entire Korean population. Eur Heart J 2019;40:809-19.

19. Kim D, Yang PS, Jang $E$, et al. Increasing trends in hospital care burden of atrial fibrillation in Korea, 2006 through 2015. Heart 2018;104:2010-17.

20. Kim D, Yang PS, Yu HT, et al. Risk of dementia in stroke-free patients diagnosed with atrial fibrillation: data from a population-based cohort. Eur Heart J 2019;40: 2313-2323.
21. Desai RJ, Franklin JM. Alternative approaches for confounding adjustment in observational studies using weighting based on the propensity score: a primer for practitioners. BM 2019;367:15657.

22. Selmer C, Hansen ML, Olesen JB, et al. New-onset atrial fibrillation is a predictor of subsequent hyperthyroidism: a nationwide cohort study. PLoS One 2013;8:e57893.

23. Dekkers OM, Horvath-Puho E, Cannegieter SC, et al. Acute cardiovascular events and all-cause mortality in patients with hyperthyroidism: a population-based cohort study. Eur J Endocrinol 2017;176:1-9.

24. Andrade JG, Aguilar M, Atzema C, et al. The 2020 Canadian Cardiovascular Society/Canadian Heart Rhythm Society Comprehensive Guidelines for the Management of Atrial Fibrillation. Can J Cardiol 2020;36:1847-948.

25. Simone JV, Abildgaard CF, Schulman I. Blood coagulation in thyroid dysfunction. N Engl J Med 1965;273:1057-61.

26. Franchini $M$. Hemostatic changes in thyroid diseases: haemostasis and thrombosis. Hematology 2006;1 1:203-8.

27. Bassand JP, Accetta G, Camm AJ, et al. 2-year outcomes of patients with newly diagnosed atrial fibrillation: results from GARFIELD-AF. Eur Heart J 2016;37:2882-9.

28. Van Zaane B, Squizzato A, Debeij J, et al. Alterations in coagulation and fibrinolysis after levothyroxine exposure in healthy volunteers: a controlled randomized crossover study. J Thromb Haemost 2011 ; 9:1816-24.

29. Collet TH, Gussekloo J, Bauer DC, et al. Subclinical hyperthyroidism and the risk of coronary heart disease and mortality. Arch Intern Med 2012;172:799-809.

30. Lillevang-Johansen M, Abrahamsen B, Jorgensen HL, et al. Duration of hyperthyroidism and lack of sufficient treatment are associated with increased cardiovascular risk. Thyroid 2019;29:332-40.

31. Osman F, Franklyn JA, Holder RL, et al. Cardiovascular manifestations of hyperthyroidism before and after antithyroid therapy: a matched case-control study. J Am Coll Cardiol 2007;49:71-81.

32. Kim TH, Yang PS, Uhm JS, et al. CHA2DS2-VASc Score (Congestive Heart Failure, Hypertension, Age >/=75 [Doubled], Diabetes Mellitus, Prior Stroke or Transient Ischemic Attack [Doubled], Vascular Disease, Age 65-74, Female) for stroke in asian patients with atrial fibrillation: a korean nationwide sample cohort study. Stroke 2017;48:1524-30. 